\title{
Heat Transfer Intensification Using Particles Across Length Scales
}

\author{
Yulong Ding \\ University of Birmingham, UK
}

\section{Extended Abstract}

This talk concerns with heat transfer intensification using particles across length scales. More specifically, three case studies:

- Particle laden flow and heat transfer in confined geometry;

- Rheological and heat transfer of dilute suspensions of nanoparticles;

- Microstructures and heat transfer of composite phase change materials

Will be used to illustrate the particle length scale effects on heat transfer. An attempt will be made to link properties of materials at particle scale to device and system level behaviour. 